\title{
SPIN MANIFOLDS, EINSTEIN METRICS, AND DIFFERENTIAL TOPOLOGY
}

\author{
Masashi Ishida And Claude LeBrun*
}

\begin{abstract}
We show that there exist smooth, simply connected, four-dimensional spin manifolds which do not admit Einstein metrics, but nevertheless satisfy the strict Hitchin-Thorpe inequality. Our construction makes use of the Bauer/Furuta cohomotopy refinement of the Seiberg-Witten invariant $[4,3]$, in conjunction with curvature estimates previously proved by the second author [17]. These methods also allow one to easily construct many examples of topological 4-manifolds which admit an Einstein metric for one smooth structure, but which have infinitely many other smooth structures for which no Einstein metric can exist.
\end{abstract}

\section{Introduction}

A smooth Riemannian metric $g$ is said to be Einstein if its Ricci curvature, considered as a function on the unit tangent bundle, is constant. As recently as the 1960's, it could have seemed reasonable to hope that every smooth compact simply connected manifold might admit such a metric; and, indeed, it was apparently with this goal in mind that Yamabe [27] carried out his trail-blazing work on the total scalar curvature. In the late 1960's, however, Thorpe [25] observed, as a parenthetical remark, that a compact oriented 4-dimensional Einstein manifold $\left(M^{4}, g\right)$ must satisfy the inequality

$$
2 \chi(M) \geq 3|\tau(M)|,
$$

where $\chi$ denotes the Euler characteristic and $\tau$ denotes the signature. This inequality was rediscovered several years later by Hitchin [12], who went on to give examples of simply connected 4-dimensional manifolds which violate inequality (1), and thus do not admit Einstein metrics. In light of this, (1) has come to be known as the Hitchin-Thorpe inequality [5].

Perhaps the deepest results in Hitchin's paper concern the boundary case of inequality (1); for it was shown in [12] that a simply connected 4-dimensional Einstein manifold with $2 \chi=-3 \tau$ must be Ricci-flat, Kähler, and diffeomorphic to the Kummer surface $K 3$. In particular, this implies that most simply connected 4-manifolds satisfying $2 \chi=3|\tau|$ do not admit Einstein metrics; and this applies not only to examples of the 'wrong' homotopy type - e.g. $\mathbb{C P}_{2} \# 9 \overline{\mathbb{C P}}_{2}$ - but also $[19,13]$ to an infinite class of manifolds $[9,7]$ now known to be

Received July 14, 2001.

Revised version received March 28, 2002.

*Supported in part by NSF grant DMS-0072591. 
homeomorphic but not diffeomorphic to $K 3$. Since Yau [28] showed that $K 3$ actually admits Einstein metrics of the kind described in Hitchin's result, this shows, in particular, that the existence of Einstein metrics on a 4-manifold is a matter of diffeotype, and not just of homeotype.

By contrast, for simply connected 4-manifolds satisfying the so-called strict Hitchin-Thorpe inequality

$$
2 \chi(M)>3|\tau(M)|
$$

obstructions to the existence of Einstein metrics remained unknown until 1995, when the second author [15] used scalar curvature estimates derived from the Seiberg-Witten equations to display an infinite class of 4-manifolds which do not admit Einstein metrics, but nonetheless satisfy (2). However, the examples given there were non-minimal complex surfaces of general type, and thus, in particular, were all non-spin. Even though these results have been subsequently improved [17] via the introduction of Weyl-curvature terms into the estimates, the non-spin character of the examples seemed to reflect an essential feature of the construction. This made it natural to wonder about the following:

Question 1. Are there simply connected 4-dimensional spin manifolds which do not admit Einstein metrics, but which nonetheless satisfy the strict HitchinThorpe inequality (2)?

In Theorem 9 below, we will see that the answer is affirmative. Our construction depends crucially on a recent breakthrough in Seiberg-Witten theory, due to Furuta and Bauer $[4,3]$, as well as on some recent curvature estimates, due to the second author [17].

Another curious defect of the examples constructed in $[15,16,17]$ is that, when oriented so that the signature is negative, all have the the property that $b_{+}$is odd. This merely reflects the fact that these examples are all almostcomplex, and the integrality of the Todd genus implies that $b_{+}$must be odd for any simply connected almost-complex 4-manifold. However, the Einstein condition is independent of orientation, and these examples can certainly have $b_{-}$of either parity. Might it not be reasonable to hope that this geographical curiosity was merely an artifact of the construction?

Question 2. Are there simply connected 4-manifolds, with $\tau<0$ and $b_{+}$even, which do not admit Einstein metrics, but which nonetheless satisfy the strict Hitchin-Thorpe inequality (2)?

In Theorem 11 below, we will see that the present methods allow one to construct such examples in considerable abundance.

Finally, we return to the heavy dependence of these questions on the choice of smooth structure. As already noted, while $K 3$ admits a smooth structure for which Einstein metrics exist, it also admits infinitely many smooth structures for which Einstein metrics do not exist. Is this typical? 
Question 3. Let $M$ be a compact simply connected topological 4-manifold which admits at least one smooth structure. Are there always infinitely many distinct smooth structures on $M$ for which no compatible Einstein metric exists?

While we are certainly not able to answer this question in full generality, we are, in Theorems 9, 11 and 12, at least able to give an affirmative answer for infinitely many homeotypes satisfying (2). Interestingly, many of these examples also have the property that, like $K 3$, they also admit smooth structures for which compatible Einstein metrics $d o$ exist. For some earlier results and ruminations in this direction, see [13].

The infinite-fundamental-group analogue of Question 3 would still appear to be a topic worthy of investigation. By contrast, however, the infinitefundamental-group analogues of Questions 1 and 2 are susceptible to the homotopy-theoretic volume estimates pioneered by Gromov, and affirmative answers $[11,21]$ to the corresponding questions have therefore already been known for some time.

\section{Monopole Classes and Curvature}

If $M$ is a smooth oriented 4-manifold, we can always find Hermitian line bundles $L \rightarrow M$ such that $c_{1}(L) \equiv w_{2}(T M) \bmod 2$. For any such $L$, and any Riemannian metric $g$ on $M$, one can then find rank-2 Hermitian vector bundles $\mathbb{V}_{ \pm}$which formally satisfy

$$
\mathbb{V}_{ \pm}=\mathbb{S}_{ \pm} \otimes L^{1 / 2}
$$

where $\mathbb{S}_{ \pm}$are the locally defined left- and right-handed spinor bundles of $(M, g)$. Such a choice of $\mathbb{V}_{ \pm}$, up to isomorphism, is called a $\operatorname{spin}^{c}$ structure $\mathfrak{c}$ on $M$, and is determined, modulo the 2-torsion subgroup of $H_{1}(M, \mathbb{Z})$, by the first Chern class $c_{1}(L)=c_{1}\left(\mathbb{V}_{ \pm}\right) \in H^{2}(M, \mathbb{Z})$ of the $\operatorname{spin}^{c}$ structure. Because $\mathbb{S}_{+}$is a quaternionic line bundle, there is a canonical anti-linear isomorphism

$$
\begin{aligned}
\mathbb{V}_{+} & \longrightarrow \mathbb{V}_{+} \otimes L^{*} \\
\Phi & \mapsto \bar{\Phi}
\end{aligned}
$$

called 'complex conjugation.' More importantly, every unitary connection $A$ on $L$ induces a Dirac operator

$$
D_{A}: \Gamma\left(\mathbb{V}_{+}\right) \rightarrow \Gamma\left(\mathbb{V}_{-}\right)
$$

If $A$ is such a connection, and if $\Phi$ is a section of $\mathbb{V}_{+}$, the pair $(\Phi, A)$ is said to satisfy the Seiberg-Witten equations [26] if

$$
\begin{aligned}
D_{A} \Phi & =0 \\
F_{A}^{+} & =-\frac{1}{2} \Phi \odot \bar{\Phi} .
\end{aligned}
$$

Here $F_{A}^{+}$is the self-dual part of the curvature of $A$, and we have identified $\Lambda^{+} \otimes \mathbb{C}$ with $\left[\odot^{2} \mathbb{V}_{+}\right] \otimes L^{*}=\odot^{2} \mathbb{S}_{+}$in the canonical manner. 
For the 4-manifolds of primary interest here, there turn out to be certain $\operatorname{spin}^{c}$ structures for which there exists a solution of the Seiberg-Witten equations for each metric $g$. This situation is neatly codified by the following terminology [14]:

Definition 1. Let $M$ be a smooth compact oriented 4-manifold with $b_{+} \geq 2$. An element $a \in H^{2}(M, \mathbb{Z}) /$ torsion will be called a monopole class of $M$ iff there exists a spin ${ }^{c}$ structure $\mathfrak{c}$ on $M$ with first Chern class

$$
c_{1}(L) \equiv a \bmod \text { torsion }
$$

which has the property that the corresponding Seiberg-Witten equations (3-4) have a solution for every Riemannian metric $g$ on $M$.

Because the Seiberg-Witten equations imply a uniform a priori bound on $\left|F_{A}^{+}\right|$ for any given metric on $g$ on $M$, it follows [26] that the set $\mathfrak{C}$ of monopole classes of any 4 -manifold $M$ is necessarily finite. Also note that $a \in \mathfrak{C} \Longleftrightarrow(-a) \in \mathfrak{C}$, since complex conjugation sends solutions of the Seiberg-Witten equations to solutions of the Seiberg-Witten equations. Finally, notice that, for any $a, b \in \mathfrak{C}$, $a-b$ is automatically divisible by 2 in the lattice $H^{2}(M, \mathbb{Z}) /$ torsion, since the corresponding first Chern classes are both $\equiv w_{2} \bmod 2$. With these observations in mind, we will now introduce a crude but effective diffeomorphism invariant of 4-manifolds.

Definition 2. Let $M$ be a smooth compact oriented 4-manifold with $b_{+}(M) \geq 2$. Let $\mathfrak{C} \subset H^{2}(M, \mathbb{Z}) /$ torsion be the set of monopole classes of $M$. If $\mathfrak{C}$ contains a non-zero element (and hence at least two elements), we define the bandwidth of $M$ to be

$$
\mathcal{B W}(M)=\max \left\{n \in \mathbb{Z}^{+} \mid \exists a, b \in \mathfrak{C}, a \neq b \text {, s.t. } 2 n \mid(a-b)\right\} .
$$

If, on the other hand, $\mathfrak{C} \subset\{0\}$, we define the bandwidth $\mathcal{B W}(M)$ to be 0 .

As was first pointed out by Witten [26], the existence of a monopole class implies an a priori lower bound on the $L^{2}$ norm of the scalar curvature of Riemannian metrics. More recently, the second author then discovered [16, 17] that (3-4) also imply a family of analogous estimates involving the self-dual Weyl curvature. For our present purposes, the most useful of these estimates is the following [17]:

Proposition 4 (LeBrun). Let $M$ be a smooth compact oriented 4-manifold with monopole class $a \in H^{2}(M, \mathbb{Z}) /$ torsion $\subset H^{2}(M, \mathbb{R})$. Let $g$ be any Riemannian metric on $M$, and let $a^{+} \in H^{2}(M, \mathbb{R})$ be the self-dual part of a with respect to the 'Hodge' decomposition

$$
H^{2}(M, \mathbb{R})=\mathcal{H}_{g}^{+} \oplus \mathcal{H}_{g}^{-}
$$

of second de Rham cohomology, identified with the space of $g$-harmonic 2-forms, into eigenspaces of the $\star$ operator. Then the scalar curvature $s$ and self-dual Weyl curvature $W_{+}$of $g$ satisfy

$$
\frac{1}{4 \pi^{2}} \int_{M}\left(\frac{s^{2}}{24}+2\left|W_{+}\right|^{2}\right) d \mu \geq \frac{2}{3}\left(a^{+}\right)^{2}
$$


where $d \mu$ denotes the Riemannian volume form of $g$, and where the point-wise norms are calculated with respect to $g$. Moreover, if $a^{+} \neq 0$, and if $a$ is not the first Chern class of a symplectic structure on $M$, then the inequality is necessarily strict.

\section{The Bauer-Furuta Invariant}

The usual Seiberg-Witten invariant [24, 18] of a smooth compact oriented 4-manifold with $b_{+} \geq 2$, equipped with some fixed $\operatorname{spin}^{c}$ structure $\mathfrak{c}$, is obtained considering the moduli space of solutions of (3-4), for a generic metric $g$, as a homology class in the configuration space

$$
\mathcal{B}=\left(\left[\Gamma\left(\mathbb{V}_{+}\right)-0\right] \times\{\text { smooth unitary connections on } L\}\right) / \mathcal{G},
$$

where $\mathcal{G}=\left\{u: M \stackrel{C^{\infty}}{\longrightarrow} S^{1}\right\}$. However, one may instead think of this moduli space as defining a framed bordism class in the space $\mathcal{D} \subset \mathcal{B}$ of solutions of (3). Pursuing this idea, Furuta and Bauer $[4,3]$ were independently able to define a refinement of the Seiberg-Witten invariant which takes values in a cohomotopy group; for example, if $b_{1}(M)=0$, the invariant takes values in $\pi^{b_{+}(M)-1}\left(\mathbb{C P}_{d-1}\right)$, where $d=\left[c_{1}^{2}(L)-\tau(M)\right] / 8$. If this Bauer-Furuta stable cohomotopy invariant is non-zero, $c_{1}(L)$ is a monopole class. But, remarkably, this invariant is not killed off by the sort of connect sum operation that would eliminate the usual Seiberg-Witten invariant [3]:

Theorem 5 (Bauer). Let $X, Y$, and $Z$ be compact oriented 4-manifolds with $b_{1}=0$ and $b_{+} \equiv 3 \bmod 4$. Suppose, that $\mathfrak{c}_{X}, \mathfrak{c}_{Y}$, and $\mathfrak{c}_{Z}$ are spinc structures of almost-complex type on $X, Y$, and $Z$ for which the mod-2 Seiberg-Witten invariant is non-zero. Then the induced spinc structures $\mathfrak{c}_{X \# Y}$ and $\mathfrak{c}_{X \# Y \# Z}$ on $X \# Y$ and $X \# Y \# Z$ have non-zero Bauer-Furuta stable cohomotopy invariant.

Now, recall that a celebrated result of Taubes [23] asserts that the mod-2 Seiberg-Witten invariant is non-zero for the canonical spin ${ }^{c}$ structure of any symplectic 4-manifold. Since the non-vanishing of the Bauer-Furuta invariant forces the existence of solutions of the Seiberg-Witten equations, we immediately obtain the following consequence:

Corollary 6. Let $X, Y$, and $Z$ be compact oriented simply connected symplectic 4-manifolds with $b_{+} \equiv 3 \bmod 4$. Then, with respect to the canonical isomorphisms

$$
\begin{aligned}
H^{2}(X \# Y, \mathbb{Z}) & =H^{2}(X, \mathbb{Z}) \oplus H^{2}(Y, \mathbb{Z}) \\
H^{2}(X \# Y \# Z, \mathbb{Z}) & =H^{2}(X, \mathbb{Z}) \oplus H^{2}(Y, \mathbb{Z}) \oplus H^{2}(Z, \mathbb{Z}),
\end{aligned}
$$

the cohomology classes

$$
\begin{aligned}
\pm c_{1}(X) \pm c_{1}(Y) & \in H^{2}(X, \mathbb{Z}) \oplus H^{2}(Y, \mathbb{Z}) \\
\pm c_{1}(X) \pm c_{1}(Y) \pm c_{1}(Z) & \in H^{2}(X, \mathbb{Z}) \oplus H^{2}(Y, \mathbb{Z}) \oplus H^{2}(Z, \mathbb{Z})
\end{aligned}
$$

are monopole classes on $X \# Y$ and $X \# Y \# Z$, respectively. Here the \pm signs are arbitrary, and independent of one another. 


\section{Obstructions to Einstein Metrics}

When combined with the ideas of [17], Corollary 6 immediately implies a non-existence result for Einstein metrics.

Theorem 7. Let $X, Y$, and $Z$ be simply connected symplectic 4-manifolds with $b_{+} \equiv 3 \bmod 4$. Then $X \# Y \# k \overline{\mathbb{C P}}_{2}$ does not admit Einstein metrics if

$$
k+4 \geq \frac{c_{1}^{2}(X)+c_{1}^{2}(Y)}{3} .
$$

Similarly, $X \# Y \# Z \# k \overline{\mathbb{C P}}_{2}$ does not admit Einstein metrics if

$$
k+8 \geq \frac{c_{1}^{2}(X)+c_{1}^{2}(Y)+c_{1}^{2}(Z)}{3} .
$$

Proof. We begin by making the identification

$$
\begin{aligned}
H^{2}\left(X \# k \overline{\mathbb{C P}}_{2}, \mathbb{Z}\right) & =H^{2}(X, \mathbb{Z}) \oplus \bigoplus_{j=1}^{k} H^{2}\left(\overline{\mathbb{C P}}_{2}, \mathbb{Z}\right) \\
& =H^{2}(X, \mathbb{Z}) \oplus \mathbb{Z}^{\oplus k}
\end{aligned}
$$

in the process making a choice of generators $E_{j}, j=1, \ldots, k$ for the $k$ copies of $H^{2}\left(\overline{\mathbb{C P}}_{2}, \mathbb{Z}\right) \cong \mathbb{Z}$. Now recall that there are self-diffeomorphisms of $X \# k \overline{\mathbb{C P}}_{2}$ which act trivially on $H^{2}(X, \mathbb{Z})$, but for which

$$
E_{j} \mapsto \pm E_{j}
$$

for any desired choice of signs. Thinking of $X \# k \overline{\mathbb{C P}}_{2}$ as the $k$-fold symplectic blow-up of $X$, and then moving the symplectic structure via these diffeomorphism, we thus obtain $2^{k}$ distinct symplectic structures on $X \# k \overline{\mathbb{C P}}_{2}$, with first Chern classes

$$
c_{1}=c_{1}(X)+\sum_{j=1}^{k}\left( \pm E_{j}\right)
$$

for all possible choices of signs. Applying Corollary 6 to $\left(X \# k \overline{\mathbb{C P}}_{2}\right) \# Y$ and $\left(X \# k \overline{\mathbb{C P}}_{2}\right) \# Y \# Z$, we thus conclude that all the classes of the form $\alpha+\sum_{j=1}^{k}\left( \pm E_{j}\right)$ are monopole classes, where

$$
\alpha= \begin{cases}c_{1}(X)+c_{1}(Y), & \text { if } M=X \# Y \# k \overline{\mathbb{C P}}_{2}, \\ c_{1}(X)+c_{1}(Y)+c_{1}(Z), & \text { if } M=X \# Y \# Z \# k \overline{\mathbb{C P}}_{2} .\end{cases}
$$

Now, given any particular metric $g$ on $M$, let us make a new choice $\hat{E}_{j}= \pm E_{j}$ of generators for our $k$ copies of $H^{2}\left(\overline{\mathbb{C P}}_{2}, \mathbb{Z}\right)$ in such a way that

$$
\hat{E}_{j} \cdot \alpha^{+} \geq 0 \text {. }
$$

The resulting monopole class

$$
a=\alpha+\sum_{j=1}^{k} \hat{E}_{j}
$$


then satisfies

$$
\begin{aligned}
\left(a^{+}\right)^{2} & =\left(\alpha^{+}+\sum \hat{E}_{j}^{+}\right)^{2} \\
& =\left(\alpha^{+}\right)^{2}+2 \sum \alpha^{+} \cdot \hat{E}_{j}^{+}+\left(\sum \hat{E}_{j}^{+}\right)^{2} \\
& \geq\left(\alpha^{+}\right)^{2} \\
& \geq \alpha^{2}
\end{aligned}
$$

Proposition 4 therefore tells us that any metric $g$ on $M$ satisfies

$$
\frac{1}{4 \pi^{2}} \int_{M}\left(\frac{s^{2}}{24}+2\left|W_{+}\right|^{2}\right) d \mu>\frac{2}{3} \alpha^{2}
$$

(The inequality is strict because $a^{2}>(2 \chi+3 \tau)(M)$, and this guarantees that $a$ is certainly not the first Chern class of a symplectic structure.)

Now for any metric $g$ on our compact orientable 4-manifold $M$, we have the Gauss-Bonnet type formula [5, 12]

$$
(2 \chi+3 \tau)(M)=\frac{1}{4 \pi^{2}} \int_{M}\left(\frac{s^{2}}{24}+2\left|W_{+}\right|^{2}-\frac{|\stackrel{\circ}{r}|^{2}}{2}\right) d \mu
$$

where $\stackrel{\circ}{r}=r-\frac{s}{4} g$ is the traceless Ricci tensor. If $g$ is Einstein, $\stackrel{\circ}{r}=0$, and inequality (5) then becomes

$$
(2 \chi+3 \tau)(M)>\frac{2}{3} \alpha^{2}
$$

For $M=X \# Y \# k \overline{\mathbb{C P}}_{2}$, we have

$$
\alpha^{2}=c_{1}^{2}(X)+c_{1}^{2}(Y)
$$

and

$$
(2 \chi+3 \tau)(M)=c_{1}^{2}(X)+c_{1}^{2}(Y)-4-k .
$$

Inequality (6) therefore asserts that a necessary condition for the existence of an Einstein metric on $M$ is that

$$
c_{1}^{2}(X)+c_{1}^{2}(Y)-4-k>\frac{2}{3}\left[c_{1}^{2}(X)+c_{1}^{2}(Y)\right],
$$

or, in other words, that

$$
\frac{c_{1}^{2}(X)+c_{1}^{2}(Y)}{3}>k+4 .
$$

By contraposition, this shows that there cannot be an Einstein metric if

$$
k+4 \geq \frac{c_{1}^{2}(X)+c_{1}^{2}(Y)}{3},
$$

exactly as claimed.

For $M=X \# Y \# Z \# k \overline{\mathbb{C P}}_{2}$, we instead have

$$
\alpha^{2}=c_{1}^{2}(X)+c_{1}^{2}(Y)+c_{1}^{2}(Z),
$$


and

$$
(2 \chi+3 \tau)(M)=c_{1}^{2}(X)+c_{1}^{2}(Y)+c_{1}^{2}(Z)-8-k,
$$

so that inequality (6) instead tells us that a necessary condition for the existence of an Einstein metric on $M$ is that

$$
c_{1}^{2}(X)+c_{1}^{2}(Y)+c_{1}^{2}(Z)-8-k>\frac{2}{3}\left[c_{1}^{2}(X)+c_{1}^{2}(Y)+c_{1}^{2}(Z)\right],
$$

or in other words that

$$
\frac{c_{1}^{2}(X)+c_{1}^{2}(Y)+c_{1}^{2}(Z)}{3}>k+8
$$

We thus conclude that there cannot be an Einstein metric if

$$
k+8 \geq \frac{c_{1}^{2}(X)+c_{1}^{2}(Y)+c_{1}^{2}(Z)}{3},
$$

and this finishes the proof.

In particular, setting $k=0$ yields:

Corollary 8. Let $X, Y$, and $Z$ be simply connected symplectic 4-manifolds with $b_{+} \equiv 3 \bmod 4$. If $c_{1}^{2}(X)+c_{1}^{2}(Y) \leq 12$, then $X \# Y$ does not admit Einstein metrics. Similarly, if $c_{1}^{2}(X)+c_{1}^{2}(Y)+c_{1}^{2}(Z) \leq 24$, then $X \# Y \# Z$ does not admit Einstein metrics.

\section{Spin Examples}

In this section, we construct a sequence of 4-dimensional spin manifolds which do not admit Einstein metrics, but nonetheless satisfy (2). Not only will we construct examples with an infinite number of homeotypes, but we will also show that each of these homeotypes carries an an infinite number of distinct smooth structures with the desired property.

We begin with a collection of symplectic spin manifolds constructed by Gompf [10]. For arbitrary integers $k \geq 2$ and $m \geq 0$, Gompf showed that one can construct, by symplectic surgery methods, a simply connected symplectic spin manifold with $(\chi, \tau)=(24 k+4 m,-16 k)$. In particular, setting $m=2$, there is a simply connected symplectic spin 4-manifold $X_{k}$ with $(\chi, \tau)=(24 k+8,-16 k)$. By the Minkowski-Hasse classification of quadratic forms, this manifold thus has intersection form $-2 k \mathbf{E}_{8} \oplus(4 k+3) \mathbf{H}$, and so is homeomorphic to $k(K 3) \#(k+$ $3)\left(S^{2} \times S^{2}\right)$ by the Freedman's theorem [8]. Notice that $b_{+}\left(X_{k}\right)=4 k+3 \equiv$ $3 \bmod 4$, and that $c_{1}^{2}\left(X_{k}\right)=(2 \chi+3 \tau)\left(X_{k}\right)=16$.

It is perhaps worth remarking that most of these symplectic manifolds cannot be taken to be complex surfaces, as most violate the Noether inequality $c_{1}^{2} \geq$ $b_{+}-5$. On the other hand, we are free e.g. to take $X_{4}$ to be a smooth complex hypersurface of tridegree $(4,4,2)$ in $\mathbb{C P}_{1} \times \mathbb{C P}_{1} \times \mathbb{C P}_{1}$. In any case, it merely suffices for what follows that we choose one such symplectic manifold $X_{k}$ for each $k \geq 2$.

The other ingredient we will need is a certain sequence of homotopy $K 3$ surfaces. Let $Y_{0}$ denote a Kummer surface, equipped with an elliptic fibration 
$Y_{0} \rightarrow \mathbb{C P}_{1}$. Let $Y_{\ell}$ be obtained from $Y_{0}$ by performing a logarithmic transformation of order $2 \ell+1$ on one non-singular elliptic fiber of $Y_{0}$. The $Y_{\ell}$ are simply connected spin manifolds with $b_{+}=3$ and $b_{-}=19$, and hence are homeomorphic to $K 3$ by the Freedman classification. However, $Y_{\ell}$ is a Kähler surface with $p_{g}=1 \neq 0$, and so, for each $\ell, \pm c_{1}\left(Y_{\ell}\right)$ are Seiberg-Witten basic class, with Seiberg-Witten invariant \pm 1 . Moreover, $c_{1}\left(Y_{\ell}\right)=2 \ell \mathfrak{f}$, where $\mathfrak{f}$ is Poincaré dual to the multiple fiber introduced by the logarithmic transformation [2].

With these building blocks in hand, we now proceed to prove the following result:

Theorem 9. For any integer $n \geq 4$, the topological spin manifold

$$
n(K 3) \#(n+1)\left(S^{2} \times S^{2}\right)
$$

admits infinitely many distinct smooth structures for which there cannot exist compatible Einstein metrics. Moreover, this topological manifold has

$$
\begin{aligned}
& \chi=24 n+4, \\
& \tau=-16 n,
\end{aligned}
$$

and thus satisfies the strict Hitchin-Thorpe inequality $2 \chi>3|\tau|$.

Proof. Set $k=n-2$, and consider the simply connected 4-manifolds

$$
M_{k, \ell}=X_{k} \# Y_{0} \# Y_{\ell} .
$$

Each of these smooth oriented 4-manifolds is homeomorphic to

$$
(k+2)(K 3) \#(k+3)\left(S^{2} \times S^{2}\right) .
$$

However, since $b_{+}\left(X_{k}\right)=4 k+3$ and $b_{+}\left(Y_{0}\right)=b_{+}\left(Y_{\ell}\right)=3$ all reduce to $3 \bmod 4$, and since

$$
c_{1}^{2}\left(X_{k}\right)+c_{1}^{2}\left(Y_{0}\right)+c_{1}^{2}\left(Y_{\ell}\right)=16+0+0<24,
$$

Corollary 8 asserts that none of these smooth manifolds $M_{k, \ell}$ can admit an Einstein metric.

Now, for any fixed $k$, observe that the sequence $\left\{M_{k, \ell} \mid \ell \in \mathbb{N}\right\}$ must contain infinitely many different diffeotypes. Indeed, Corollary 6 asserts that $a=c_{1}\left(X_{k}\right)+c_{1}\left(Y_{\ell}\right)$ and $b=c_{1}\left(X_{k}\right)-c_{1}\left(Y_{\ell}\right)$ are both monopole classes on $M_{k, \ell}=X_{k} \# Y_{0} \# Y_{\ell}$. However, the difference $a-b=2 c_{1}\left(Y_{\ell}\right)$ is divisible by $4 \ell$, and the bandwidth of $M_{k, \ell}$, as defined in Definition 2, therefore satisfies

$$
\mathcal{B W}\left(M_{k, \ell}\right) \geq 2 \ell \text {. }
$$

Thus, for any fixed $k$,

$$
\sup _{\ell \in \mathbb{N}} \mathcal{B} \mathcal{W}\left(M_{k, \ell}\right)=\infty
$$

and it follows that no individual $M_{k, \ell}$ has maximal bandwidth, for any given $k$. Hence $\left\{M_{k, \ell} \mid \ell \in \mathbb{N}\right\}$ runs through infinitely many different diffeotypes for each $k$, and the claim follows. 


\section{Non-Spin Examples}

We conclude this article by showing that Theorem 7 also provides a rich source of non-spin 4-manifolds without Einstein metrics.

We will begin with a sequence of simply connected symplectic manifolds constructed by Gompf [10]. Namely, for each integer $i \geq 2$, there is a compact simply connected symplectic 4 -manifold $Z_{i}$ with Todd genus $\frac{1}{4}(\chi+\tau)\left(Z_{i}\right)=i$ and $c_{1}^{2}\left(Z_{i}\right)=8 i-11$. The easiest thing to do with these examples is to apply the results of [17], whereby one gets non-existence of Einstein metrics on $Z_{i} \# k \overline{\mathbb{C P}}_{2}$ for $k \geq(8 i-11) / 3$. Since the resulting manifolds are simply connected and nonspin, with $b_{+}=2 i-1$ and $b_{-}=2 i+10+k$, Freedman's theorem [8] immediately yields

Proposition 10. Let $(m, n)$ be a pair of natural numbers, where $m$ is odd. If $n>\frac{7}{3} m+8$, there is a smooth structure on $m \mathbb{C P}_{2} \# n \overline{\mathbb{C P}}_{2}$ for which there is no compatible Einstein metric.

Note that these examples satisfy the strict Hitchin-Thorpe inequality (2) provided that we also require that $n<5 m+4$; such examples therefore exist for any $m \neq 1$. Moreover, many of these examples are actually homeomorphic to Einstein manifolds; cf. $[13,16,17]$. Of course, this result does not pretend to be optimal; e.g. sporadic improvements could certainly be made by exploiting the manifolds constructed by Stipsicz [22].

A much more interesting result is obtained, however, by applying Theorem 7 to $Z_{2 j} \# Y_{\ell} \# k \overline{\mathbb{C P}}_{2}$, where the $Y_{\ell}$ are the homotopy $K 3$ surfaces used in the previous section. If $k>\frac{16}{3} j-8$, these manifolds do not admit Einstein metrics. On the other hand, for fixed $j, k$, the same reasoning used in the proof of Theorem 9 shows that

$$
\lim _{\ell \rightarrow \infty} \mathcal{B W}\left(Z_{2 j} \# Y_{\ell} \# k \overline{\mathbb{C P}}_{2}\right)=\infty,
$$

so we obtain infinitely many distinct smooth structures for each topological type. This proves the following:

Theorem 11. Let $(m, n)$ be a pair of natural numbers with $m \equiv 2 \bmod 4$ and $m \geq 6$. If $n>\frac{7}{3} m+16$, there are infinitely many distinct smooth structure on $m \mathbb{C P}_{2} \# n \overline{\mathbb{C P}}_{2}$ for which no compatible Einstein metric exists.

Again, these manifolds will satisfy the strict Hitchin-Thorpe inequality if $n<$ $5 m+4$, and such examples thus occur for each allowed value of $m$.

One can also apply the same method to $Z_{2 j} \# R_{2,2} \# Y_{\ell} \# k \overline{\mathbb{C P}}_{2}$, where $R_{2,2}$ is the simply connected symplectic manifold constructed by Gompf [10], with $b_{+}=3$ and $b_{-}=14$, and where $k \geq \frac{16}{3} j-10$. By the same bandwidth argument, we obtain

Theorem 12. Let $(m, n)$ be a pair of natural numbers with $m \equiv 1 \bmod 4$ and $m \geq 9$. If $n>\frac{7}{3} m+12$, there are infinitely many distinct smooth structure on $m \mathbb{C P}_{2} \# n \overline{\mathbb{C P}}_{2}$ for which no compatible Einstein metric exists. 
For each allowed value of $m$, we once again obtain examples satisfying the strict Hitchin-Thorpe inequality. But indeed, many of these topological manifolds are known to admit some other smooth structure for which a compatible Einstein metric does exist.

Example. For any integer $p \geq 6$ with $p \equiv 2 \bmod 4$, set $m=p^{2}-3 p+3$ and $n=3 p^{2}-3 p+1$. We then have $m \equiv 1 \bmod 4, m \geq 9$, and $n>\frac{7}{3} m+12$; thus, Theorem 12 therefore asserts that there are infinitely many smooth structures on $m \mathbb{C P}_{2} \# n \overline{\mathbb{C P}}_{2}$ for which no Einstein metrics exists. However, this homeotype is also realized by a double branched cover of $\mathbb{C P}_{2}$, ramified over a smooth curve of degree $2 p$. The latter complex surface of general type contains no $(-1)$ - or $(-2)$-curves, and so has ample canonical line bundle [2]; the Aubin/Yau theorem $[1,28]$ therefore tells us that it admits an Einstein (Kähler) metric. The situation is thus analogous to that of the $K 3$ surface; for one smooth structure, there is an Einstein metric, but for infinitely many others, no such metric exists.

Of course, the $K 3$ surface has the remarkable additional property that it admits only one differentiable structure for which there exists a compatible Einstein metric. In light of [20], however, no such uniqueness statement holds for many of the topological manifolds under discussion; cf. [6, 13]. Nonetheless, one might speculate that Einstein metrics can exist only for a finite number of smooth structures on any given topological 4-manifold. Perhaps this difficult question will become a fruitful topic for future research.

\section{Acknowledgment}

We would like to express our deep gratitude to Professors Stefan Bauer and Mikio Furuta for helping us come to grips with the key features of the BauerFuruta invariant.

\section{References}

[1] T. Aubin, Equations du type Monge-Ampère sur les variétés kählériennes compactes, C. R. Acad. Sci. Paris Sér. A-B 283 (1976), no. 3, Aiii, A119-A121.

[2] W. Barth, C. Peters, and A. V. de Ven, Compact complex surfaces, Ergebnisse der Mathematik und ihrer Grenzgebiete (3), 4. Springer-Verlag, Berlin, 1984.

[3] S. Bauer, A stable cohomotopy refinement of Seiberg-Witten invariants. II, preprint, 2001. Posted at http://xxx.lanl.gov/abs/math.DG/0204267

[4] S. Bauer and M. Furuta, A stable cohomotopy refinement of Seiberg-Witten invariants. I, preprint, 2001. Posted at http://arXiv.org/abs/math/0204340

[5] A. Besse, Einstein Manifolds, Ergebnisse der Mathematik und ihrer Grenzgebiete (3), 10. Springer-Verlag, Berlin, 1987.

[6] F. Catanese and C. LeBrun, On the scalar curvature of Einstein manifolds, Math. Res. Lett. 4 (1997), no. 6, 843-854.

[7] R. Fintushel and R. J. Stern, Constructions of smooth 4-manifolds, Proceedings of the International Congress of Mathematicians, Vol. II (Berlin, 1998). Doc. Math. 1998, Extra Vol. II, 443-452 (electronic).

[8] M. Freedman, The topology of four-dimensional manifolds, J. Differential Geom. 17 (1982), no. 3, 357-453. 
[9] R. Friedman and J. W. Morgan, On the diffeomorphism types of certain algebraic surfaces. I, J. Differential Geom. 27 (1988), no. 2, 297-369.

[10] R. E. Gompf, A new construction of symplectic manifolds, Ann. of Math. (2) 142 (1995), no. 3, 527-595.

[11] M. Gromov, Volume and bounded cohomology, Inst. Hautes Études Sci. Publ. Math. No. 56, (1982), 5-99 (1983).

[12] N. Hitchin, Compact four-dimensional Einstein manifolds, J. Differential Geom. 9 (1974), 435-441.

[13] D. Kotschick, Einstein metrics and smooth structures, Geom. Topol. 2 (1998), 1-10 (electronic).

[14] P. B. Kronheimer, Minimal genus in $S^{1} \times M^{3}$, Invent. Math. 135 (1999), no. 1, 45-61.

[15] C. LeBrun, Four-manifolds without Einstein metrics, Math. Res. Lett. 3 (1996), no. 2, $133-147$.

[16] Weyl curvature, Einstein metrics, and Seiberg-Witten theory, Math. Res. Lett. 5 (1998), no. 4, 423-438.

[17] _ Ricci curvature, minimal volumes, and Seiberg-Witten theory, Invent. Math. 145 (2001), no. 2, 279-316.

[18] P. Ozsváth and Z. Szabó, Higher type adjunction inequalities in Seiberg-Witten theory, J. Differential Geom. 55 (2000), no. 3, 385-440.

[19] Y.-S. Poon, private communication, 1995. Posted, by permission of the author, at http://www.math. sunysb.edu/ ${ }^{\sim}$ claude/sun

[20] M. Salvetti, On the number of nonequivalent differentiable structures on 4-manifolds, Manuscripta Math. 63 (1989), no. 2, 157-171.

[21] A. Sambusetti, An obstruction to the existence of Einstein metrics on 4-manifolds, C. R. Acad. Sci. Paris Sér. I Math. 322 (1996), no. 12, 1213-1218.

[22] A. I. Stipsicz, Simply connected 4-manifolds near the Bogomolov-Miyaoka-Yau line, Math. Res. Lett. 5 (1998), no. 6, 723-730.

[23] C. H. Taubes, The Seiberg-Witten invariants and symplectic forms, Math. Res. Lett. 1 (1994), no. 6, 809-822.

[24] _ The Seiberg-Witten and Gromov invariants, Math. Res. Lett. 2 (1995), no. 2, $221-238$.

[25] J. A. Thorpe, Some remarks on the Gauss-Bonnet integral, J. Math. Mech. 18 (1969), 779-786.

[26] E. Witten, Monopoles and four-manifolds, Math. Res. Lett. 1 (1994), no. 6, 769-796.

[27] H. Yamabe, Collected works of Hidehiko Yamabe, Gordon and Breach, Science Publishers, New York-London-Paris, 1967.

[28] S.-T. Yau, Calabi's conjecture and some new results in algebraic geometry, Proc. Nat. Acad. Sci. U.S.A. 74 (1977), no. 5, 1789-1799.

Department of Mathematics, Sophia University, Kioicho, Chiyoda-ku, Tokyo 1028554, JAPAN.

E-mail address: ishida@m.sophia.ac.jp

Department of Mathematics, SUny Stony Brook, Stony Brook, Ny 11794-3651, U.S.A.

E-mail address: claude@math.sunysb.edu 